\title{
Biliary tract perforation following percutaneous endobiliary radiofrequency ablation: A report of two cases
}

\author{
CHUANGUO ZHOU, BAOJIE WEI, KUN GAO and RENYOU ZHAI \\ Department of Interventional Radiology, Beijing Chaoyang Hospital, The Affiliated Hospital of Capital Medical University, \\ Beijing 100020, P.R. China
}

Received January 12, 2015; Accepted February 9, 2016

DOI: $10.3892 / \mathrm{ol} .2016 .4436$

\begin{abstract}
Endobiliary radiofrequency ablation (RFA) has recently been recognized as a beneficial treatment option for malignant biliary obstruction using percutaneous or endoscopic approaches. The feasibility and safety of this method has been demonstrated in clinical studies, with pain, cholangitis and asymptomatic biochemical pancreatitis reported as relatively common complications. By contrast, hepatic coma, newly diagnosed left bundle branch block and partial liver infarction have been reported as uncommon complications. Biliary tract perforation is a serious potential complication of percutaneous intraductal RFA, which may result in severe infection, peritonitis or even mortality, and which has not been previously reported in clinical research. The current study presents the first reports of biliary tract perforation in two patients with unresectable malignant biliary obstruction following percutaneous intraductal RFA. Although the patient in case 1 succumbed 12 days after RFA, the minor biliary tract perforation in case 2 was successfully treated by the deployment of a self-expanding metal stent. This study demonstrates that biliary tract perforation should be recognized as a serious potential complication of endobiliary RFA, and that metal stent deployment should be considered as a treatment option for minor biliary tract perforation.
\end{abstract}

\section{Introduction}

Biliary tract cancers have a median survival rate of 4.8 months with a 1- and 5-year survival rate of 31 and $10 \%$ respectively (1). A diagnosis is often made at an advanced disease stage in patients with malignant biliary obstruction (2). Such

Correspondence to: Professor Renyou Zhai, Department of Interventional Radiology, Beijing Chaoyang Hospital, The Affiliated Hospital of Capital Medical University, 8 Gongren Tiyuchang Nan Road, Beijing 100020, P.R. China

E-mail: ryzhai219@sina.com

Key words: biliary tract perforation, percutaneous endobiliary radiofrequency ablation, pancreatic carcinoma, cholangiocarcinoma, complication malignancies are known to be unresectable and have a poor prognosis. For patients with a life expectancy of $>3$ months, self-expandable metal stents are deployed (3); however, occlusion of these stents has been reported in $50 \%$ of patients within 6 months of deployment (2). The relatively high failure rate is attributed to tumor in- and overgrowth, epithelial hyperplasia, biofilm deposition, debris formation and sludge buildup. Furthermore, covered nitinol metal stents have shown an inability to increase stent patency or patient survival time (4). Photodynamic therapy (PDT) is not a common treatment option for biliary tract cancers, but it is a novel palliative therapy that may have a direct effect on the local tumor within the bile duct lumen with promising results for the maintenance of biliary drainage (3). However, the availability, photosensitivity and procedural cost of PDT is limited, and the procedure necessitates repeated treatment sessions (5). In addition, a recent multicenter, randomized phase III study demonstrated that patients that received PDT with stenting had a 61\% excess mortality risk with a poorer overall survival compared with patients that received stenting alone (3).

Recently, intraductal radiofrequency ablation (RFA) has become a beneficial therapeutic option against malignant biliary obstruction. Clinical studies by Steel et al (2) and Mizandari et al (6) demonstrated the safety of endobiliary RFA treatment, with asymptomatic biochemical pancreatitis, cholangitis and pain recognized as the only common complications; however, in the present study, RFA caused biliary tract perforation, a previously unreported complication that can result in serious infection, peritonitis and even mortality.

\section{Case report}

Case 1. A 67-year-old male presented with a biliary obstruction with symptoms that consisted of icteric scelra, fever, fatigue, chills, anorexia, nausea, vomiting and elevated blood bilirubin at Beijing Chaoyang Hospital (Beijing, China) in May 2013. Contrast-enhanced computed tomography (CT) revealed a space-occupying lesion in the extrahepatic bile duct (Fig. 1). The patient was diagnosed with cholangiocarcinoma based on the symtoms and imaging. An exploratory laparotomy was performed in May 2013 at the Cancer Hospital of Chinese Academy of Medical Sciences (Beijing, China), which revealed that it was an unresectable malignant biliary tumor. Therefore, percutaneous transhepatic biliary 

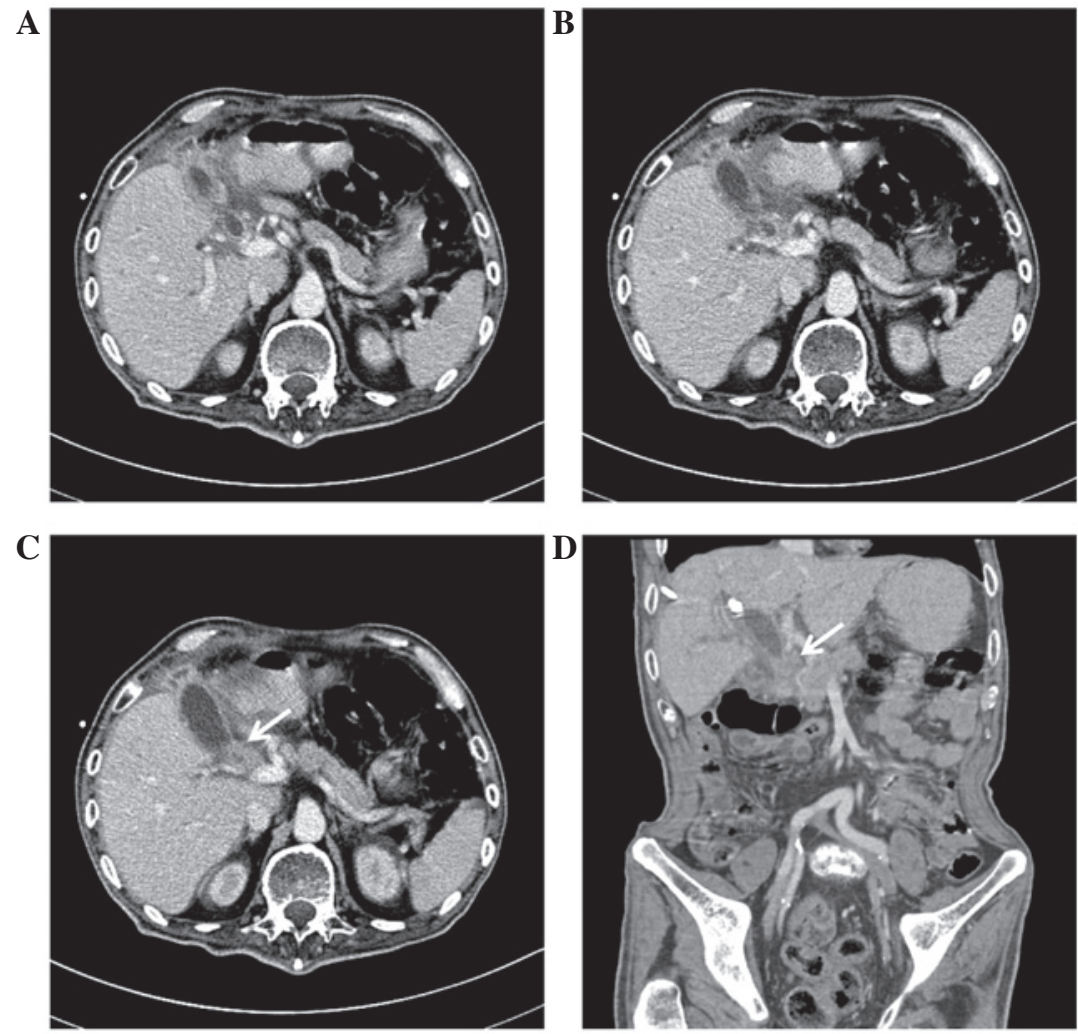

Figure 1. Case 1. Contrast-enhanced computed tomography. (A) Mild dilation of the intrahepatic bile duct was observed, with a visible upper common bile duct and invisible middle and lower ducts. (B) Migration location between the dilated upper common bile duct and the tumor. (C) A clearly enhanced, higher density space-occupying lesion was present between the gastric antrum and duodenum (arrow). (D) Coronal reconstructed image showing the dilated common bile duct. The arrow indicates the lesion location.

drainage was conducted to relieve the obstructive jaundice, which decreased the total bilirubin from 352 to $78 \mathrm{mmol} / \mathrm{l}$ (normal range, $0-20 \mathrm{mmol} / \mathrm{l}$ ).

After obtaining informed consent from the patient, percutaneous intraductal RFA was performed on December 3, 2013, using an 8 Fr percutaneous endobiliary RFA catheter (Habib; EMcision Ltd., London, UK) placed using a guidewire from the distal segment of the common bile duct to the proximal segment of the common hepatic duct (Fig. 2A-C). RFA was applied for $2 \mathrm{~min}$ at $10 \mathrm{~W}$ to two sections of stricture within the biliary duct sections with a 1-min rest period in-between. An 8x80-mm Zilver self-expanding metal stent (ZIV6-80-8-8.0; Cook Inc., Bloomington, IN, USA) was then deployed in the stenosis section of the bile duct and inflated using an 8x40-mm dilatation balloon catheter (ATB5-35-40-8-4.0; Cook Inc.) (Fig. 2D and E). Percutaneous transhepatic cholangiography (PTC) was performed in which the contrast agent overflowed to the outside of the biliary duct, confirming the diagnosis of biliary tract perforation (Fig. 2F). The patient, however, also presented with melena the following day. Despite the application of various treatments, such as blood transfusion $(1,400 \mathrm{ml})$, fluid infusion $(1,500 \mathrm{ml} /$ day; consisting of amino acids, lipid emulsion, glucose, sodium chloride, water, vitamins and potassium chloride) and anti-infection therapies (cefoxitin sodium injection, $2 \mathrm{~g} 3$ times/day for 12 days), the patient succumbed on December 15, 2013.

Case 2. A 61-year-old male with obstructive jaundice was diagnosed with pancreatic carcinoma at Beijing Chaoyang
Hospital in October 2013, based on the symptoms, laboratory findings and imaging manifestations that were exhibited by the patient. Symptoms consisted of weakness, anorexia and icteric scelra; laboratory examination revealed elevated blood bilirubin $(257 \mathrm{mmol} / \mathrm{l})$ and tumor marker cancer antigen 19-9 (2,687 U/ml; normal range, 0-39 U/ml); and an abdominal CT scan revealed a space-occupying lesion in the head of pancreas with dilation of the extra- and intrahepatic biliary duct. The patient had undergone a surgical resection for esophagocardial cancer 3 months previously at the Cancer Hospital of Chinese Academy of Medical Sciences. Due to the patient's poor physical condition [Karnofsky performance status score, 70 (7)], microinvasive treatment with interventional radiology was considered the best option. Percutaneous transhepatic biliary drainage was conducted to relieve the obstructive jaundice, which decreased the total bilirubin from 257 to $65 \mathrm{mmol} / \mathrm{l}$. PTC showed that the biliary duct stricture section was located at the common bile duct (Fig. 3A).

After obtaining informed consent from the patient, percutaneous intraductal RFA was combined with self-expandable metal stent deployment on November 28, 2013. A guidewire was used to place the 8 Fr percutaneous endobiliary RFA catheter in two sections of the common bile duct to ablate the malignant tumor. RFA was applied for $2 \mathrm{~min}$ at $10 \mathrm{~W}$ in each section following a 1-min rest period. Due to the severity of the biliary duct stenosis, an 8x40-mm dilatation balloon catheter was used to pre-expand the bile duct for stent deployment (Fig. 3B). Following the dilation, PTC showed a considerably 

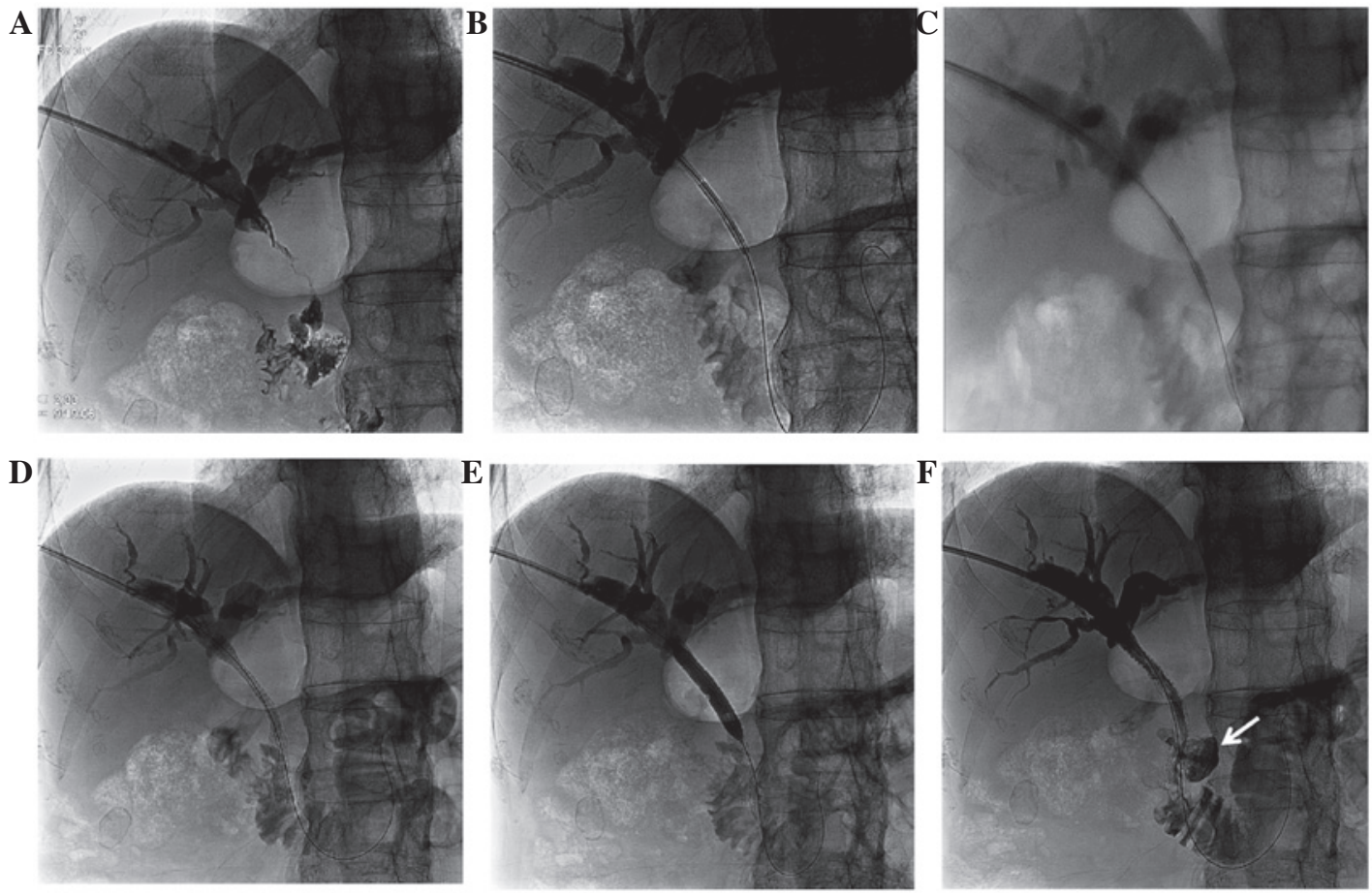

Figure 2. Case 1. Percutaneous transhepatic cholangiography. (A) Severe stenosis was observed in the common bile duct. (B and C) A percutaneous endobiliary radiofrequency ablation catheter was placed in two sections of the biliary duct stricture, from the distal segment of the common bile duct to the proximal segment of the common hepatic duct. (D) A self-expanding metal stent was deployed. (E) A dilatation balloon catheter was used to inflate the metal stent. (F) Biliary tract perforation was diagnosed based on the overflow of the contrast agent to the outside of the biliary duct (arrow).
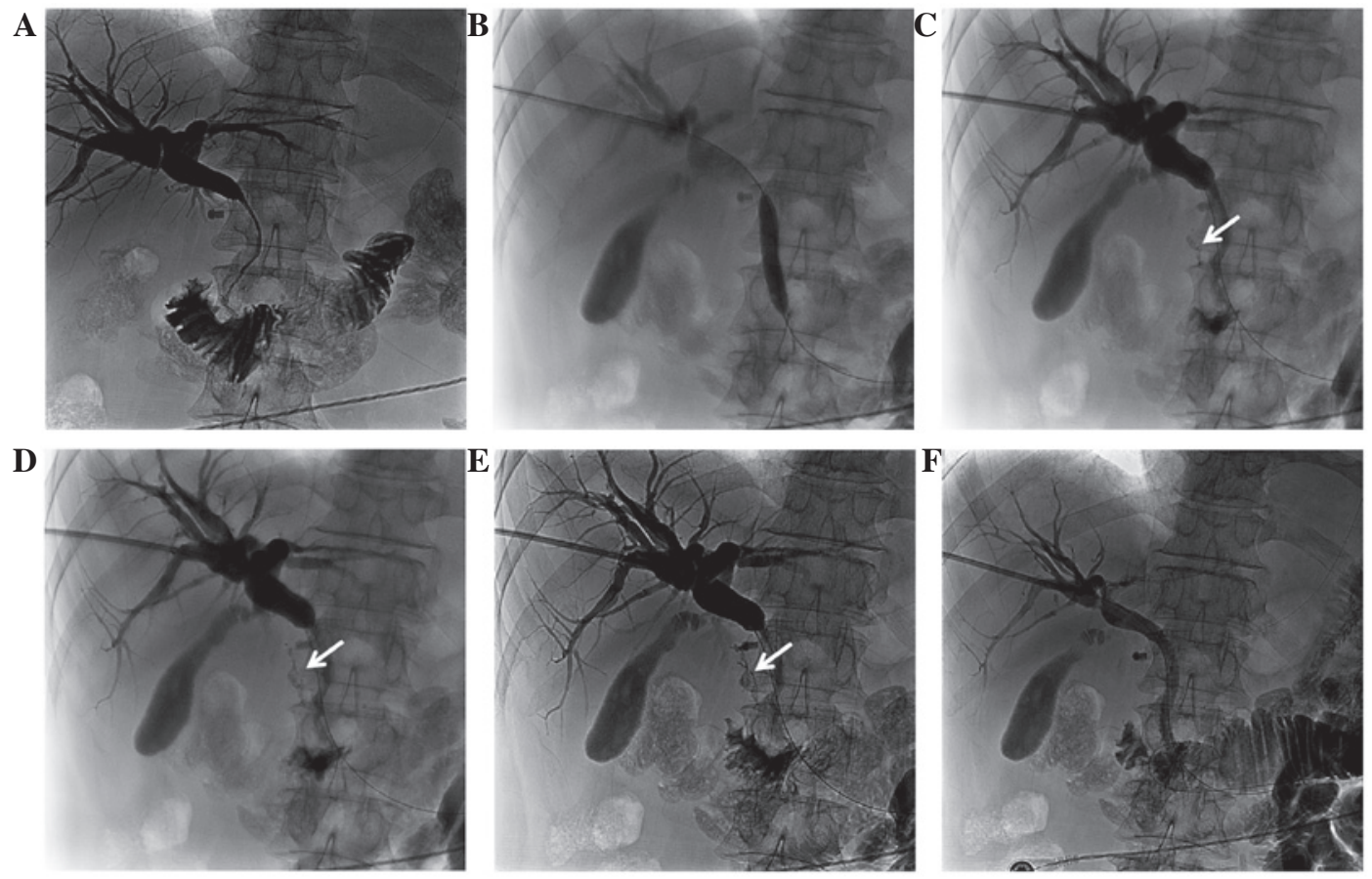

Figure 3. Case 2. Percutaneous transhepatic cholangiography. (A) Severe stenosis of the common bile duct was observed. (B) A dilatation balloon catheter was used to expand the bile duct following intraductal radiofrequency ablation. (C-E) A biliary tract perforation of considerably small diameter was diagnosed based on the overflow of the contrast agent to the outside of the biliary duct (arrows). (F) Following metal stent deployment, no overflow of the contrast agent was observed outside the bile duct.

small-diameter biliary tract perforation and overflow of the contrast agent (Fig. 3C-E), although no signs of choleperitonitis were observed. The $8 \times 80-\mathrm{mm}$ Zilver self-expanding metal stent was then deployed to cover the perforated region.
PTC following stent deployment showed no contrast agent overflow to the outside of the bile duct (Fig. 3F), and the patient was discharged 7 days later. No follow-up of the patient was performed. 


\section{Discussion}

For patients with malignant biliary strictures and a life expectancy of $>3$ months, self-expandable metal stent deployment is the standard treatment option. The addition of RFA could prove beneficial to such patients, as a recent retrospective study comparing metal stenting with and without RFA showed (8). The study demonstrated that RFA was an independent predictor of survival in the treatment of malignant biliary strictures. RFA with an image-guided minimally invasive interventional approach has been used for several years to treat patients with a variety of other primary and secondary malignancies, including hepatocellular carcinoma, lung and breast cancer, musculoskeletal system tumors, and renal and metastatic carcinomas (9). The use of an ablation catheter in malignant biliary obstruction was approved by The United States Food and Drug Administration and the EU/EC Declaration of Conformity (10); however, intraductal RFA has not been considered, due to the expected complication of thermal biliary damage from heating and direct mechanical damage from the needle, such as bile leakage or biliary stricture (11). By contrast, biliary damage is a relatively uncommon complication following RFA of hepatic tumors, with an incidence of $0.1-12.0 \%$ (12).

There have been reports of the use of intraductal RFA for the treatment of unresectable malignant biliary obstructions with percutaneous and endoscopic approaches, demonstrating its feasibility and safety; pain, cholangitis and asymptomatic biochemical pancreatitis have been reported as the only common complications $(2,6,13)$, while hepatic coma, newly diagnosed left bundle branch block and partial liver infarction have been reported as uncommon complications $(2,14,15)$. However, a few cases of hemobilia and mortality following intrabiliary RFA have also been reported (15). Despite the fact that, to the best of our knowledge, there had previously been no cases of biliary tract perforations following RFA, the said complication occurred in the two cases mentioned in the present study, which was confirmed by PTC. These cases involved unresectable malignant biliary obstructions; a cholangiocarcinoma and a pancreatic carcinoma.

In the present study, the two cases received overlapping RFA for 2 min at $10 \mathrm{~W}$. Thermal injury induced deep bile duct necrosis and possibly caused the perforations. Furthermore, the dilatation of the bile duct with a balloon catheter (prior to RFA in case 1 , and following RFA in case 2) may have aggravated the strong necrotic effect induced by RFA thermal injury. For the treatment of minor biliary tract perforation, the insertion of a self-expanding metal stent directly after the RFA procedure appears to be an effective method (case 2); however, the management of major biliary tract perforation is more challenging and may result in severe complications, including mortality (case 1). Possible preemptive strategies for the prevention of biliary tract perforation could include pre-interventional investigation with $\mathrm{CT} /$ magnetic resonance imaging, in order to identify the extent of the tumor around the bile duct and avoid injuring the normal bile duct. It is also important to avoid overlapping RFA. Finally, the use of balloon angioplasty prior to or following RFA should be carefully assessed.

The patient in case 1 also presented with melena the day after RFA, indicating the possibility of a direct association between the procedure and gastrointestinal bleeding. Only three studies have reported a similar occurrence following intrabiliary RFA, including six cases of hemobilia and one case of hepatic artery pseudoaneurysm rupture $(14,16,17)$. In the present case, endoscopy was performed to identify the bleeding site, but no injury or active bleeding was observed in the duodenum. Thermal injury to the hepatic artery was therefore presumed as the cause.

In conclusion, despite the therapeutic advantages of endobiliary RFA against jaundice and the possibility of an RFA-induced increase in the survival time of patients with unresectable malignant biliary obstructions, biliary tract perforation should be acknowledged as a serious potential complication of the procedure, and the deployment of self-expanding metal stents should be considered a preferable treatment option for minor biliary perforation.

\section{References}

1. Pinter M, Hucke F, Zielonke N, et al: Incidence and mortality trends for biliary tract cancers in Austria. Liver Int 34: 1102-1108, 2014.

2. Steel AW, Postgate AJ, Khorsandi S, et al: Endoscopically applied radiofrequency ablation appears to be safe in the treatment of malignant biliary obstruction. Gastrointest Endosc 73: 149-153, 2011.

3. Rustagi T and Jamidar PA. Intraductal radiofrequency ablation for management of malignant biliary obstruction. Dig Dis Sci 59: 2635-2641, 2014.

4. Kullman E, Frozanpor F, Söderlund C, et al: Covered versus uncovered self-expandable nitinol stents in the palliative treatment of malignant distal biliary obstruction: Results from a randomized, multicenter study. Gastrointest Endosc 72: 915-923, 2010.

5. Law R, Pai M, Baron TH and Habib N: The effects of endobiliary radiofrequency ablation in two patients with pancreatic cancer: Gross and microscopic findings. Gastrointest Interv 2: 124-126, 2013.

6. Mizandari M, Pai M, Xi F, et al: Percutaneous intraductal radiofrequency ablation is a safe treatment for malignant biliary obstruction: Feasibility and early results. Cardiovasc Intervent Radiol 36: 814-819, 2013.

7. Karnofsky DA, Abelmann WH, Craver LF and Burchenal JH: The use of nitrogen mustard in the palliative treatment of cancer with particular reference to bronchogenic carcinoma. Cancer 1: 634-656, 1948.

8. Sharaiha RZ, Natov N, Glockenberg KS, et al: Comparison of metal stenting with radiofrequency ablation versus stenting alone for treating malignant biliary strictures: Is there an added benefit? Dig Dis Sci 59: 3099-3102, 2014.

9. Gazelle GS, Goldberg SN, Solbiati L and Livraghi T: Tumor ablation with radio-frequency energy. Radiology 217: 633-646, 2000.

10. Lui KL and Li KK: Intraductal radiofrequency ablation of tumour ingrowth into an uncovered metal stent used for inoperable cholangiocarcinoma. Hong Kong Med J 19: 539-541, 2013.

11. Livraghi T, Solbiati L, Meloni MF, et al: Treatment of focal liver tumors with percutaneous radio-frequency ablation: Complications encountered in a multicenter study. Radiology 226: 441-451, 2003.

12. Fonseca AZ, Santin S, Gomes LG, Waisberg J and Ribeiro MA Jr: Complications of radiofrequency ablation of hepatic tumors: Frequency and risk factors. World J Hepatol 6: 107-113, 2014.

13. Figueroa-Barojas P, Bakhru MR, Habib NA, et al: Safety and efficacy of radiofrequency ablation in the management of unresectable bile duct and pancreatic cancer: A novel palliation technique. J Oncol 2013: 910897, 2013.

14. Dolak W, Schreiber F, Schwaighofer H, et al: Endoscopic radiofrequency ablation for malignant biliary obstruction: A nationwide retrospective study of 84 consecutive applications. Surg Endosc 28: 854-860, 2014.

15. Alis H, Sengoz C, Gonenc M, Kalayci MU and Kocatas A: Endobiliary radiofrequency ablation for malignant biliary obstruction. Hepatobiliary Pancreat Dis Int 12: 423-427, 2013

16. Tal AO, Vermehren J, Friedrich-Rust M, et al: Intraductal endoscopic radiofrequency ablation for the treatment of hilar non-resectable malignant bile duct obstruction. World J Gastrointest Endosc 6: 13-19, 2014.

17. Topazian M,Levy MJ, Patel S, Charlton MR and Baron TH: Hepatic artery pseudoaneursym formation following intraductal biliary radiofrequency ablation. Endoscopy 45 (Suppl 2): S161-S162, 2013. 